\title{
Adaptive Transmission with Discrete Code Rates and Channel State Uncertainty
}

\author{
Lang Lin, Roy D. Yates, and Predrag Spasojević \\ WINLAB, Rutgers University \\ 73 Brett Rd., NJ 08854-8060 \\ $\{1$ lin, ryates, spasojev $\} @$ winlab.rutgers.edu
}

\begin{abstract}
Without perfect channel state information at the transmitter, it is possible for adaptive transmission systems to experience information outage. In this paper, we formulate the throughput maximization with both an average power constraint and an information outage constraint. It is verified that, for the optimal transmission policy, the transmission only needs to adapt to a sufficient statistic for the channel state. For a Rayleigh fading channel with a simple training scheme, numerical results show that, with a reasonable amount of training and a small set of code rates, the adaptive transmission can achieve a performance very close to the ergodic capacity.
\end{abstract}

\section{INTRODUCTION}

In order to combat fading, adaptive transmission techniques have been considered and applied in many wireless systems. Typically, the performance of adaptive transmission techniques is evaluated based on a set of idealized assumptions, such as perfect channel estimates and a continuously varying code rate. Thus, the task of the analysis is greatly simplified. Perhaps, by relaxing idealized assumptions, one can hope to gain further insight into this problem and improve guideline for developing practical systems.

To accommodate practical constraints, an adaptive $M$ ary quadrature amplitude modulation (MQAM) with a finite number of modulation levels and an adaptive trellis-coded modulation (TCM) scheme with a finite number of code rates are proposed in [1] and [2], respectively. More recently, an adaptive transmission design based on outdated channel information and either MQAM or TCM is proposed in [3].

We are interested in the maximum achievable throughput of a system with adaptive transmission techniques. In [4], the throughput maximization of discrete code rates for adaptive transmission systems is studied. However, it is assumed that perfect channel state information (CSI) is available. In [5], throughput maximization with channel uncertainty is studied. In that paper, one of the adaptation parameters, code rate, is assumed to be a continuous variable. Here, we combine the assumptions of the previous two papers together with an additional outage constraint and study the problem of throughput maximization with both discrete code rates and channel uncertainty.

We assume a slow multiplicative fading environment with additive white Gaussian noise (AWGN). The channel response is constant during the transmission of a codeword. Perfect
CSI and a channel measurement are available at the receiver and the transmitter, respectively. The joint distribution of the channel measurement and the current channel state is assumed to be known. For each transmission, a message is encoded at a rate selected from a finite rate set based on the channel measurement and the resulting codeword is transmitted at a power level based on the same channel estimate. Since each codeword experiences an additive white Gaussian noise (AWGN) channel, random Gaussian codes with multiple codebooks are employed.

For the proposed discrete adaptive system, it is possible that the instantaneous mutual information corresponding to a channel state is less than the assigned code rate. In this case, an information outage event occurs. The information outage is an intrinsic characteristic of communication over fading channels with a decoding delay constraint [6], [7] or, alternatively, with codewords not long enough to experience ergodic fading. Moreover, it has been shown that the outage probability matches well the error probability of actual codes [8], [9]. Thus, since typical communication services must sustain a certain QoS requirement in terms of the error performance, we introduce an additional information outage constraint to the traditional power constrained throughput maximization. In this paper, we demonstrate that the posed constrained throughput maximization problem can be solved in a general case.

\section{System Model And Problem Formulation}

\section{A. System Model}

We consider a multiplicative flat fading channel model similar to that in [10]. The complex received signal

$$
Y=\sqrt{S} X+W
$$

where $S$ is the channel (fading) state, $X$ is the complex transmitted signal, and $W$ is a circularly symmetric additive white Gaussian noise (AWGN) with variance $N_{0}$. The channel state $S$ is a real random variable of unit mean with a probability density function (PDF) $f_{S}(s)$, a cumulative distribution function $(\mathrm{CDF}) F_{S}(s)$, and a domain $\mathcal{S}=\{s \mid s \geq 0\}$. It is also assumed that the fading is sufficiently slow that the channel state is constant during transmission of a codeword.

The transmission is designed in such a way that before transmitting a data message, the transmitter obtains a channel 
measurement vector $\mathbf{u}=\left[u_{0}, \ldots, u_{M-1}\right]^{\top}$, where $M$ is the number of measuments. $\mathbf{U}$ is the corresponding random vector with a domain $\mathcal{U}$. We assume that $S$ and $\mathbf{U}$ have a joint $\operatorname{PDF} f_{S, \mathbf{U}}(s, \mathbf{u})=f_{S \mid \mathbf{U}}(s \mid \mathbf{u}) f_{\mathbf{U}}(\mathbf{u})$. Our assumption is rather general. For example, in a block fading channel, $\mathbf{U}$ may be based on the observation corresponding to training symbols transmitted during a finite set of past channel states. Furthermore, measurements can be a function of the stochastic process that describes the channel state evolution.

Given a measurement $\mathbf{u}$, the transmitter selects a code rate $r(\mathbf{u})$ from $\mathcal{R}=\left\{r_{0}=0, r_{1}, \ldots, r_{L}\right\}$ and a power level $p(\mathbf{u})=E\left\{|X|^{2} \mid \mathbf{u}\right\}$, where $E\{\cdot\}$ denotes expectation, to transmit the data message. It is assumed that perfect CSI is available at the receiver side. Here, without loss of generality, we assume that $r_{l} \leq r_{l+1}$ for $l=0, \ldots, L-1$.

\section{B. Outage, Policy, and Capacity}

Since $\mathbf{u}$ is only a measurement, it is possible that the corresponding channel state does not support the assigned transmitted rate $r(\mathbf{u})$ given $p(\mathbf{u})$ and, thus, an outage occurs. The corresponding conditional outage probability is

$$
\begin{aligned}
P_{\text {out }}(\mathbf{u}) & =\operatorname{Pr}\left[\log \left(1+\frac{p(\mathbf{u}) S}{N_{0}}\right)<r(\mathbf{u})\right] \\
& =\operatorname{Pr}\left[S<\frac{N_{0}}{p(\mathbf{u})}\left(e^{r(\mathbf{u})}-1\right)\right],
\end{aligned}
$$

and the worst channel state that supports the rate $r(\mathbf{u})$ is

$$
q(\mathbf{u})=\frac{N_{0}}{p(\mathbf{u})}\left(e^{r(\mathbf{u})}-1\right) .
$$

Note that (4) is only meaningful when $p(\mathbf{u})>0$ and, thus, a meaningful $q(\mathbf{u})$ is strictly positive. Consequently,

$$
P_{\text {out }}(\mathbf{u})=F_{S \mid \mathbf{U}}(q(\mathbf{u}) \mid \mathbf{u}),
$$

where $F_{S \mid \mathbf{U}}(\cdot \mid \mathbf{u})$ is the conditional $\mathrm{CDF}^{1}$ of $S$ for any $\mathbf{u}$ given $\mathbf{U}=\mathbf{u}$ and is assumed to be strictly increasing in $s$.

An adaptive transmission policy is uniquely identified by $(p(\mathbf{u}), r(\mathbf{u}))$ or equivalently, $(p(\mathbf{u}), q(\mathbf{u}))$, where $q(\mathbf{u})$ is the worst channel state $s$ that still allows for $r(\mathbf{u})$ to be achieved given $\mathbf{u}$. The system throughput is

$$
\bar{R}(p(\cdot), q(\cdot))=\int_{\mathcal{U}} \log \left(1+\frac{p(\mathbf{u}) q(\mathbf{u})}{N_{0}}\right) f_{\mathbf{U}}(\mathbf{u}) d \mathbf{u} .
$$

The corresponding average power is

$$
\rho(p(\cdot), q(\cdot))=\int_{\mathcal{U}} p(\mathbf{u}) f_{\mathbf{U}}(\mathbf{u}) d \mathbf{u} .
$$

Since communication in a wireless system is typically power limited, we consider the average transmitted power constraint

$$
\rho(p(\cdot), q(\cdot)) \leq \bar{p} .
$$

${ }^{1} F_{S \mid \mathbf{U}}(q(\mathbf{u}) \mid \mathbf{u})=\operatorname{Pr}[S \leq q(\mathbf{u}) \mid \mathbf{U}=\mathbf{u}]$. Other conditional CDFs used in this paper are defined in the same way.
Besides (8), we also assume an equality outage constraint,

$$
P_{\text {out }}(\mathbf{u})=P_{\text {out }}, \quad \mathbf{u} \in\left\{\mathbf{u}^{\prime} \mid p\left(\mathbf{u}^{\prime}\right)>0\right\},
$$

where $P_{\text {out }}$ is the positive error probability requirement. Note that the equality (9) is a simplification of the practical QoS requirement in terms of the error performance. For instance, it is normally assumed that voice communication with an error probability up to $1 \%$ is acceptable. We will study the inequality constraint in future work.

In this paper, we try to maximize the throughput in (6) over a discrete rate set $\mathcal{R}$ and a continuously varying power allocation subject to both the average transmitted power constraint (8) and the equality outage constraint (9). The throughputmaximizing policies are referred as optimal policies.

\section{Properties of Optimal Policies}

\section{A. Local Properties}

Due to the outage constraint (9), the worst supportable channel state satisfies

$$
P_{\text {out }}=F_{S \mid \mathbf{U}}(q(\mathbf{u}) \mid \mathbf{u}), \quad \mathbf{u} \in \mathcal{U} .
$$

Within the scope of this paper, we assume that $\operatorname{Pr}[S=0 \mid \mathbf{U}=\mathbf{u}] \leq P_{\text {out }}$, for any $\mathbf{u} \in \mathcal{U}$. Thus, since $F_{S \mid \mathbf{U}}(s \mid \mathbf{u})$ is strictly increasing in $s, q(\mathbf{u})$ is uniquely specified by $P_{\text {out }}$ and $F_{S \mid \mathbf{U}}(s \mid \mathbf{u})$. Let $G_{\mathbf{u}}(s)=F_{S \mid \mathbf{U}}(s \mid \mathbf{u})$ and, then, we have

$$
q(\mathbf{u})=G_{\mathbf{u}}^{-1}\left(P_{\text {out }}\right) .
$$

Thus, with (4), $p(\mathbf{u})$ and $r(\mathbf{u})$ have a one-to-one relationship.

In order to understand the structure of our problem, we define the incremental efficiency or efficiency

$$
\begin{aligned}
\eta_{l}(\mathbf{u}) & =\frac{r_{l}-r_{l-1}}{\frac{N_{0}}{q(\mathbf{u})}\left(e^{r_{l}}-1\right)-\frac{N_{0}}{q(\mathbf{u})}\left(e^{r_{l-1}}-1\right)} \\
& =\frac{r_{l}-r_{l-1}}{\frac{N_{0}}{q(\mathbf{u})}\left(e^{r_{l}}-e^{r_{l-1}}\right)} \\
& =\left(\frac{r_{l}-r_{l-1}}{e^{r_{l}-r_{l-1}}-1}\right) \frac{q(\mathbf{u})}{e^{r_{l-1} N_{0}}},
\end{aligned}
$$

where $l=1, \ldots, L$. The efficiency is the ratio of the rate increment of the adjacent rates over the corresponding increment in the transmitted power required to sustain the rate increment.

Proposition 1 The efficiency has the following properties.

1) $\eta_{l}(\mathbf{u})$ is non-negative;

2) $\eta_{l}(\mathbf{u})$ decreases in $l$.

Due to limited space, the proofs for all propositions and theorems are omitted. From Proposition 1, it is clear that we pay a heavier penalty in terms of power to transmit at a higher rate regardless of observations. Consequently, it is intuitive that reducing the average power dictates that lower rates should be transmitted. 


\section{B. Global Properties}

We define the most power efficient allocation (MPEA) as a policy with $r(\mathbf{u})=r_{l}$ if and only if $\eta_{l}(\mathbf{u}) \geq \lambda$ and $\eta_{l+1}(\mathbf{u})<$ $\lambda$ for some $\lambda>0$ and $l=1, \ldots, L$.

Theorem 1 For some $\lambda>0$, MPEA is an optimum policy.

Corollary 1 The optimal policies have the same rate/power allocation at $\mathbf{u}_{1}$ and $\mathbf{u}_{2}$ if $f_{S \mid \mathbf{U}}\left(s \mid \mathbf{u}_{1}\right) \equiv f_{S \mid \mathbf{U}}\left(s \mid \mathbf{u}_{2}\right)$.

Let $T(\mathbf{u})$ be a sufficient statistic for $s$. Then [11],

$$
f_{\mathbf{U} \mid S}(\mathbf{u} \mid s)=f_{T(\mathbf{U}) \mid S}(T(\mathbf{u}) \mid s) h(\mathbf{u}), \quad h(\mathbf{u}) \geq 0,
$$

where $h(\mathbf{u})$ is a deterministic function which is independent of $s$ and

$$
f_{S \mid \mathbf{U}}(s \mid \mathbf{u})=\frac{f_{T(\mathbf{U}) \mid S}(T(\mathbf{u}) \mid s) f_{S}(s)}{\int_{0}^{\infty} f_{T(\mathbf{U}) \mid S}(T(\mathbf{u}) \mid s) f_{S}(s) d s} .
$$

Corollary 1 and (16) imply that the optimal policies have

$$
r\left(\mathbf{u}_{1}\right)=r\left(\mathbf{u}_{2}\right), \quad p\left(\mathbf{u}_{1}\right)=p\left(\mathbf{u}_{2}\right),
$$

for any measurements $\mathbf{u}_{1}$ and $\mathbf{u}_{2}$ whenever $T\left(\mathbf{u}_{1}\right)=T\left(\mathbf{u}_{2}\right)$. Therefore, in the rest of this paper, we will concentrate on rate/power allocations over values of $T(\mathbf{U})$ instead of $\mathbf{U}$. In particular, let

$$
V=T(\mathbf{U}),
$$

where $V$ is a random variable with a domain $\mathcal{V}$. It is sufficient to specify policies of interest by $(r(v), p(v))$, where $r(v)$ and $p(v)$ are the rate and power allocations corresponding to any measurement $\mathbf{u}$ satisfying $v=T(\mathbf{u})$. Equivalently, $v$ indicates a set of measurements, $\{\mathbf{u} \mid T(\mathbf{u})=v\}$ which share the same rate $r(v)$ and power $p(v)$.

Accordingly, the following functions are re-defined accordingly,

$$
\begin{aligned}
q(v) & =\frac{N_{0}}{p(v)}\left(e^{r(v)}-1\right) \\
\bar{R}(p(\cdot), q(\cdot)) & =\int_{\mathcal{V}} \log \left(1+\frac{p(v) q(v)}{N_{0}}\right) f_{V}(v) d v \\
\rho(p(\cdot), q(\cdot)) & =\int_{\mathcal{V}} p(v) f_{V}(v) d v \\
\eta_{l}(v) & =\left(\frac{r_{l}-r_{l-1}}{e^{r_{l}-r_{l-1}}-1}\right) \frac{q(v)}{e^{r_{l-1} N_{0}}}
\end{aligned}
$$

We assume that the upper limit of $\mathcal{V}$ is the same as that of $\mathcal{S}$ which is infinity.

Let $\mathcal{V}_{l}=\left\{v \mid r(v)=r_{l}\right\}, \quad l=0, \ldots, L$, be a nonoverlapping partition of $\mathcal{V}$. Then, the average power allocated over $\mathcal{V}_{l}$ is

$$
\begin{aligned}
P_{l} & =\int_{\mathcal{V}_{l}} p(v) f_{l}(v) d v \\
& =\int_{\mathcal{V}_{l}} \frac{N_{0}}{q(v)}\left(e^{r_{l}}-1\right) f_{l}(v) d v \\
& =\frac{N_{0}}{\omega_{l}}\left(e^{r_{l}}-1\right) .
\end{aligned}
$$

where

$$
\begin{aligned}
\frac{1}{\omega_{l}} & =\int_{\mathcal{V}_{l}} \frac{1}{q(v)} f_{l}(v) d v, \\
f_{l}(v) & =\frac{f_{V}(v)}{F_{l}} \\
F_{l} & =\int_{\mathcal{V}_{l}} f_{V}(v) d v .
\end{aligned}
$$

Note that $\omega_{l}$ can be regarded as the average channel quality.

Clearly, if $\mathcal{V}_{l}$ is known, an optimal policy solves

$$
\begin{array}{cc} 
& \max _{P_{l}} \sum_{l=1}^{L} \log \left(1+\frac{P_{l} \omega_{l}}{N_{0}}\right) F_{l} \\
\text { subject to } \quad & \sum_{l=1}^{L} P_{l} F_{l} \leq \bar{p} .
\end{array}
$$

The optimization (29) is identical to the well-known rate maximization in the parallel Gaussian channel [12] and the following theorem can be obtained by directly applying the Karush-Kuhn-Tucker conditions.

Theorem 2 The optimal power allocation is in a water-filling form satisfying

$$
P_{l}=N_{0}\left[K_{0}-\frac{1}{\omega_{l}}\right]^{+},
$$

where $K_{0}$ is a constant ensuring the power constraint $\sum_{l=1}^{L} P_{l} F_{l}=\bar{p}$. The corresponding optimal rates are

$$
r_{l}=\log \left(1+\left[K_{0} \omega_{l}-1\right]^{+}\right) .
$$

In addition, it can be shown that the allocated power is zero only for $v \in \mathcal{V}_{0}$. This means that if the water-filling allocation results $P_{l}=0$ for $l>0$, an inefficient set of rates $\mathcal{R}$ has been chosen.

\section{Proposition 2 For optimal policies,}

$$
P_{l}>0, \quad l=1, \ldots, L,
$$

and

$$
r_{l}=\log \left(K_{0} \omega_{l}\right)
$$

where

$$
K_{0}=\frac{\bar{p} / N_{0}+\sum_{l=1}^{L} F_{l} / \omega_{l}}{\sum_{l=1}^{L} F_{l}} .
$$

\section{Sufficient Statistic with Stochastic Ordering}

In this subsection, we concentrate on a special class of problems with such a sufficient statistic $V$ that $F_{S \mid V}(s \mid v)$ is a collection of distributions with stochastic ordering [13]. Moreover, we concentrate on the case of stochastically increasing scenarios where $v<v^{\prime}$ implies $F_{S \mid V}(s \mid v)>F_{S \mid V}\left(s \mid v^{\prime}\right)$ for all $s \in \mathcal{S}$. It states that the channel state $s$ corresponding to $v<v^{\prime}$ is statistically worse than that corresponding to $v^{\prime}$. 
In this paper, the outage probability is in the form of the conditional distribution of $s$ given $v$ and we proceed to explore the significance that stochastic ordering brings.

Proposition 3 The efficiency corresponding to a sufficient statistic $v$ satisfies the following.

1) $\eta_{l}(v)$ is non-negative;

2) $\eta_{l}(v)$ decreases in $l$;

3) $\eta_{l}(v)$ increases in $v$.

The third condition of Proposition 3 implies the following proposition.

Proposition 4 The optimal $\mathcal{V}_{l}$ 's are

$$
\mathcal{V}_{l}=\left[v_{l}, v_{l+1}\right), \quad l=0, \ldots, L-1,
$$

where $v_{L+1}=\infty$ and $v_{l}<v_{l+1}, l=0, \ldots, L$.

Following Proposition 2 and Proposition 4, the policy of interest can uniquely be specified by $(\mathbf{r}, \mathbf{v})$, where

$$
\begin{aligned}
\mathbf{r} & =\left\{r_{1}, \ldots, r_{L}\right\}, \\
\mathbf{v} & =\left\{v_{1}, \ldots, v_{L}\right\}
\end{aligned}
$$

The corresponding throughput and the average power are

$$
\begin{aligned}
R(\mathbf{r}, \mathbf{v}) & =\sum_{l=1}^{L} r_{l} \int_{\mathcal{V}_{l}} f_{V}(v) d v \\
\rho(\mathbf{r}, \mathbf{v}) & =\sum_{l=1}^{L}\left(e^{r_{l}}-1\right) \int_{\mathcal{V}_{l}} \frac{N_{0}}{q(v)} f_{V}(v) d v .
\end{aligned}
$$

A necessary condition for the optimal policies follows as

$$
\eta_{l}(v)=\left(\frac{r_{l}-r_{l-1}}{e^{r_{l}}-e^{r_{l-1}}}\right) \frac{q\left(v_{l}\right)}{N_{0}}=\frac{1}{N_{0} K_{0}},
$$

where

$$
K_{0}=\frac{\bar{p} / N_{0}+\int_{v_{1}}^{\infty} \frac{1}{q(v)} f_{V}(v) d v}{\int_{v_{1}}^{\infty} f_{V}(v) d v},
$$

follows from (35). Note that (41) is meaningful since $q(v)$ of interest is positive.

Since $\frac{r_{l}-r_{l-1}}{e^{r_{l}}-e^{m l-1}}$ decreases in $r_{l}$, according to (41), we can find a unique $r_{l}$ given $K_{0}, r_{l-1}$, and $q\left(v_{l}\right)$. Consequently, a unique $r_{l}$ can be found given $K_{0}, r_{l-1}$, and $v_{l}$. On the other hand, (34) guarantees that a unique $\omega_{l}$ given $r_{l}$. Together with (26), $v_{l+1}$ can be uniquely specified by $r_{l}$ and $v_{l}$ since $q(v)$ is strictly positive. Thus, an iterative algorithm can be constructed to find the optimal policy starting from $v_{1}$.

Theorem 3 An optimal policy can be uniquely specified by $v_{1}$.

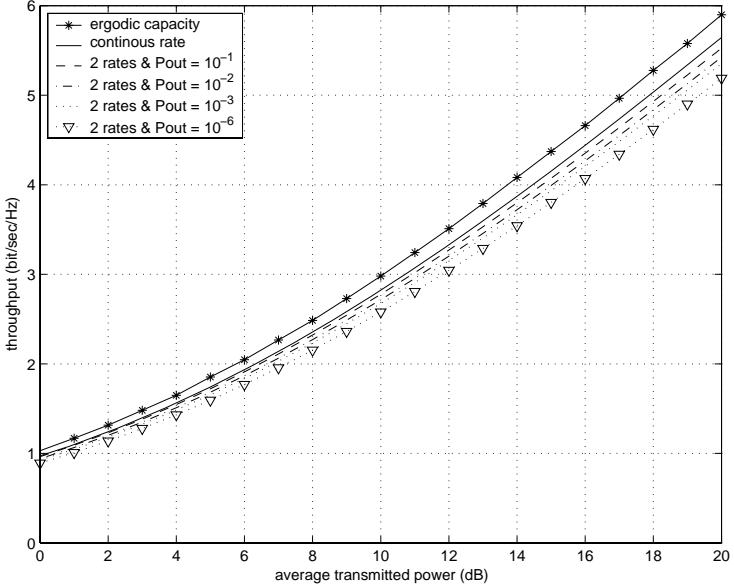

Fig. 1. Sample performance in a Rayleigh fading channel with $\gamma_{\mathrm{T}}=1000$.

\section{TRAining BASED AdAPTIVE TRANSMission}

The transmission is designed in such a manner that, before transmitting a data message, the channel is probed by a training sequence consisting of $M$ identical symbols with an amplitude $x_{\mathrm{T}}=\sqrt{\mathcal{E}_{\mathrm{T}}}$. Note that $\mathcal{E}_{\mathrm{T}}$ is the transmitted energy corresponding to a training symbol. Based on the corresponding $M$ received symbols $\mathbf{u}=\left[u_{0}, \ldots, u_{M-1}\right]^{\top}$, the transmitter selects a code rate $r(\mathbf{u})$ to encode and a power level $p(\mathbf{u})$ to transmit the data message. The signal-to-noise ratio (SNR) for the training sequence is

$$
\gamma_{\mathrm{T}}=M \frac{E\left\{\mathcal{E}_{\mathrm{T}} S\right\}}{N_{0}} .
$$

For simplicity, we assume that the training symbols experience the same channel $s$ as the transmitted information (payload) (1). In addition, we assume that the phase of the received signals is accurately known at the receiver. In this case,

$$
f_{\mathbf{U} \mid S}(\mathbf{u} \mid s)=\left(\pi N_{0}\right)^{-M} \exp \left\{-\frac{\left\|\mathbf{u}-\sqrt{s} \mathbf{x}_{\mathrm{T}}\right\|^{2}}{N_{0}}\right\},
$$

where

$$
\mathbf{x}_{\mathrm{T}}=\left[x_{\mathrm{T}}, \ldots, x_{\mathrm{T}}\right] .
$$

A sufficient statistic for $s$ given $\mathbf{u}$ is

$$
T(\mathbf{u})=\Re\left(\mathbf{u}^{\dagger} \mathbf{x}_{\mathrm{T}}\right) .
$$

Hence, with (18), we have

$$
f_{V \mid S}(v \mid s)=\left(\pi M \mathcal{E}_{\mathrm{T}} N_{0}\right)^{-\frac{1}{2}} \exp \left[-\frac{\left(v-M \mathcal{E}_{\mathrm{T}} \sqrt{s}\right)^{2}}{M \mathcal{E}_{\mathrm{T}} N_{0}}\right] .
$$

For a Rayleigh fading channel with

$$
f_{S}(s)=e^{-s},
$$


we have

$$
\begin{gathered}
f_{S, V}(s, v)=c_{a}^{-\frac{1}{2}}\left[\pi N_{0} c_{b}\right]^{-\frac{1}{2}} \exp \left[\frac{\left(c_{a} c_{b}^{2}-1\right) v^{2}}{N_{0} M \mathcal{E}_{\mathrm{T}}}\right] \\
\exp \left[-\frac{\left(\sqrt{s}-c_{b} v\right)^{2}}{N_{0} c_{b}}\right]
\end{gathered}
$$

where $c_{a}=M \mathcal{E}_{\mathrm{T}}\left(M \mathcal{E}_{\mathrm{T}}+N_{0}\right)$ and $c_{b}=M \mathcal{E}_{\mathrm{T}} / c_{a}$.

Then, we can find $f_{V}(v)^{2}$ and $F_{S \mid V}(q \mid v)^{3}$. It is straightforward to verify that $V$ is a sufficient statistic satisfying the stochastic ordering requirement.

\section{SAMPLE RESUlT}

In Fig. 1, we present a sample numerical result for a Rayleigh fading channel. Here, following [14], we let $N_{0}=1$ in order to evaluate the system performance numerically.

The ergodic capacity curve is reproduced according to [10]. For the rest of curves, we fix $\gamma_{\mathrm{T}}=1000$ or $30 \mathrm{~dB}$. When there is an infinite number of rate levels $(L=\infty)$, the throughput is within $1 \mathrm{~dB}$ from the ergodic capacity.

The last four curves correspond to $L=2$ and various $P_{\text {out }}$ 's. From these curves, we can see that reducing $P_{\text {out }}$ makes the constraint more stringent and, thus, lowers the overall throughput.

In Fig. 2, it is shown that within a wide range of $\gamma_{\mathrm{T}}$, the results with $L=2$ are very close to those with $L=\infty$.

\section{REFERENCES}

[1] A. Goldsmith and S. Chua, "Variable-rate variable-power MQAM for fading channels," IEEE Transactions on Communications, vol. 45, no. 10, pp. 1218-1229, Oct. 1997.

[2] A. Goldsmith and S. Chua, "Adaptive coded modulation for fading channels," IEEE Transactions on Communications, vol. 46, no. 5, pp. 595-602, May 1998.

[3] D. Goeckel, "Adaptive coding for time-varying channels using outdated fading estimates," IEEE Transactions on Communications, vol. 47, no. 6, pp. 844-855, Jun. 1999.

[4] L. Lin, R. Yates, and P. Spasojevic, "Adaptive transmission with discrete rates," IEEE Proceedings of ICC'02, vol. 7, pp. 2261-2265, June 2002.

[5] L. Lin, R. Yates, and P. Spasojevic, "Adaptive transmission with channel uncertainty," IEEE Proceedings of ISIT'02, p. 109, July 2002.

[6] L. Ozarow, S. Shamai, and A. Wyner, "Information theoretic considerations for cellular mobile radio," IEEE Transactions on Vehicular Technology, vol. 43, no. 2, pp. 359-378, May 1994.

[7] S. Hanly and D. Tse, "Multi-access fading channels: Part II: Delaylimited capacities," IEEE Transactions on Information Theory, vol. 44, no. 7, pp. 2816-2831, Nov. 1998.

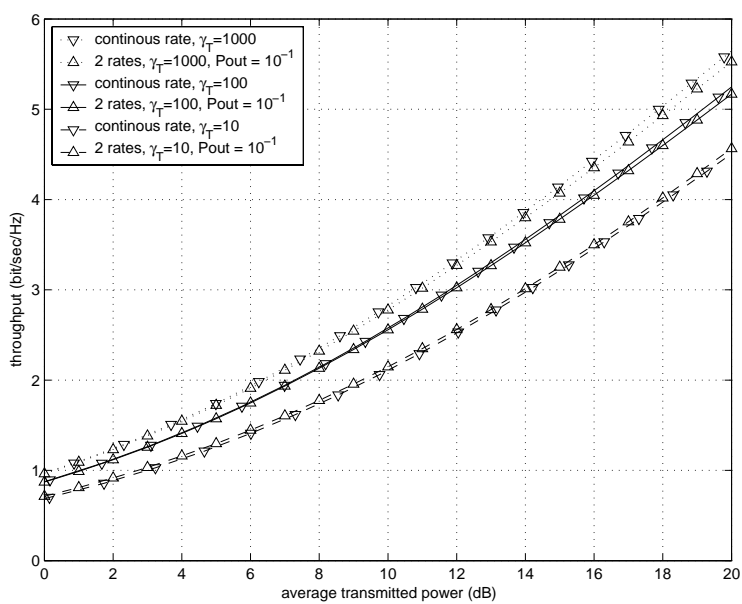

Fig. 2. Sample performance in a Rayleigh fading channel with variable $\tau_{T}$.

[8] R. Knopp and P. Humblet, "On coding for block fading channels," IEEE Transactions on Information Theory, vol. 46, no. 1, pp. 189-205, Jan. 2000.

[9] E. Malkamäki and H. Leib, "Coded diversity on block-fading channels," IEEE Transactions on Information Theory, vol. 45, no. 2, pp. 771-781, Mar. 1999.

[10] A. Goldsmith and P. Varaiya, "Capacity of fading channels with channel side information," IEEE Transactions on Information Theory, vol. 43 , no. 6, pp. 1986-1992, Nov. 1997.

[11] H. L. Van Trees, Detection Estimation and Modulation Theory, vol. 1-3, Wiley and Sons, 1971.

[12] T. Cover and J. Thomas, Elements of Information Theory, Johe Wiley \& Sons, Inc., 1991

[13] E. Lehmann, Testing Statistical Hypotheses (2nd Edition), SpringerVerlag, 1997.

[14] G. Caire, G. Taricco, and E. Biglieri, "Optimum power control over fading channels," IEEE Transactions on Information Theory, vol. 45, no. 5 , pp. $1468-1489$, July 1999.

$f_{V}(v)=c_{a}^{-\frac{1}{2}} \exp \left[\frac{\left(c_{a} c_{b}^{2}-1\right) v^{2}}{N_{0} M \mathcal{E}_{\mathrm{T}}}\right]\left[c_{b} v \operatorname{erfc}\left(-\sqrt{\frac{c_{b}}{N_{0}}} v\right)+\sqrt{\frac{N_{0} c_{b}}{\pi}} \exp \left(-\frac{c_{b}}{N_{0}} v^{2}\right)\right]$,

where

$$
\operatorname{erfc}(\phi)=\frac{2}{\sqrt{\pi}} \int_{\phi}^{\infty} e^{-t^{2}} d t
$$

$1-F_{S \mid V}(q \mid v)=\frac{\sqrt{c_{b}} v \operatorname{erfc}\left(\sqrt{\frac{q}{N_{0} c_{b}}}-\sqrt{\frac{c_{b}}{N_{0}}} v\right)+\sqrt{\frac{N_{0}}{\pi}} \exp \left(-\left(\sqrt{\frac{q}{N_{0} c_{b}}}-\sqrt{\frac{c_{b}}{N_{0}}} v\right)^{2}\right)}{\sqrt{c_{b}} v \operatorname{erfc}\left(-\sqrt{\frac{c_{b}}{N_{0}}} v\right)+\sqrt{\frac{N_{0}}{\pi}} \exp \left(-\frac{c_{b}}{N_{0}} v^{2}\right)}$. 